\title{
Clinically oriented contour evaluation using geometric and dosimetric indices based on geometric transformation
}

\section{Lixun Xian}

West China Hospital, Sichuan University

\section{Guangjun Li}

West China Hospital, Sichuan University

Qing Xiao

West China Hospital, Sichuan University

Zhibin Li

West China Hospital, Sichuan University

Xiangbin Zhang

West China Hospital, Sichuan University

Li Chen

West China Hospital, Sichuan University

Zhenyao Hu

West China Hospital, Sichuan University

Sen Bai ( $\sim$ baisen@scu.edu.cn )

\section{Research}

Keywords: Contour evaluation, Geometric indices, Dosimetric indices, Geometric transformation simulation

Posted Date: March 25th, 2020

DOI: https://doi.org/10.21203/rs.3.rs-19265/v1

License: (c) (1) This work is licensed under a Creative Commons Attribution 4.0 International License.

Read Full License 


\section{Abstract}

Background : In radiotherapy, geometric indices are often used to evaluate the accuracy of contouring; However, the ability of geometric indices to identify the error of contouring results is limited, and there is lack of clinical background. Based on the reference contouring, we systematically introduced geometric errors to study the relationship between geometric and dosimetric indices and evaluated the clinical feasibility of assessing the accuracy of contouring based on geometric indices alone.

Materials and Methods: A C-shaped target, organ at risk (Core), and intensity-modulated radiotherapy (IMRT) plan outlined in the American Association of Physicists in Medicine (AAPM) TG-119 report (The report of Task Group 119 of the AAPM) were used as references. Translation, scaling, rotation (except for the Core), and sine function transformation were performed to simulate the contouring results. The corresponding dosimetric indices were obtained from the original dose distribution of the radiotherapy plan, and correlations $\left(\mathrm{R}^{2}\right)$ between geometric indices and dosimetric indices were quantified through linear regression. The clinical applicability of the threshold for geometric indices was analyzed by combining the geometric indices and dose difference diagram.

Results: The correlations between the geometric and dosimetric indices were different and inconsistent for the contouring of the target and Core after the geometric transformation simulation. Except for the sine function transformation ( $\left.R^{2}: 0.04-0.023, p>0.05\right)$, the other three geometric indices of the planning target volume (PTV) had strong correlations with the dosimetric indices $D 98 \%$ and $D$ mean $\left(R^{2}: 0.689\right.$ 0.988), $80 \%$ of which were strongly correlated with a $p<0.001$. The correlation results for the other geometric transformations in the Core were similar to those in the PTV except for the down shift transformation.

Conclusions: The dosimetric indices are heavily influenced by the contour differences, thus highlighting their importance in the evaluation process. Clinically, an assessment of the contour accuracy of the region of interest is not feasible based on geometric indices alone, and should be combined with dosimetric indices. Keywords: Contour evaluation, Geometric indices, Dosimetric indices, Geometric transformation simulation

\section{Background}

Contouring of the target and organ at risk (OAR) is a key step in radiotherapy, especially with the highly modulated radiotherapy technology currently in use, such as intensity-modulated radiotherapy (IMRT) and volumetric modulated arc radiotherapy (VMAT). A problem with this step will cause serious system errors [1, 2]. The commonly used slice-by-slice manual approach or interpolation-based semi-automatic contouring approach are time-consuming and resource-consuming, and the corresponding results are susceptible to differences between observers; furthermore, the accuracy of contouring can also be affected by the experience of residents and the quality of computed tomography (CT) images [3-6]. Another disadvantage is that the four-dimensional (4D) imaging technology used to match the breathing 
movements can add uncertainty to the contouring of the region of interest (ROI) [7]. It is important to ensure the accuracy of contouring.

Methods for assessing the accuracy of contouring are generally divided into two categories, namely, subjective evaluations and quantitative evaluations. Subjective evaluations refer to the ROI contoured by experts as reference contours, and then, data are compared and scored with respect to the reference data [8]. This evaluation method is deeply affected by the individual differences among the evaluators and required considerable time; therefore, most contour accuracy studies are performed directly by using quantitative evaluation methods, which involves the employment of geometric indices to characterize the accuracy of the contouring results. Geometric indices widely used in contour evaluations include distance-type geometric indices (e.g., the maximum (HD), mean $\left(\mathrm{HD}_{\text {mean }}\right)$, and $95 \%$ Hausdorff distances (HD95)) and volumetric geometric indices (e.g., Dice-similarity coefficient (DSC) and Jaccard) [9]. Although these indices are easy to calculate, they do not consider the clinical effect and may lack clinical relevance [10,11]; additionally, there is considerable variability between them [12-14]. Under the assumption of a reference contour, the method for clinically assessing the accuracy of radiotherapy (RT) contours is to determine and predict the deviation of its dosimetric indices based on the dose distribution of the radiation treatment plan $[10,15,16]$. However, the relationships between geometric indices and dosimetric indices have not been investigated thoroughly.

At present, there are many methods for contouring the ROI. For the accuracy of the contouring, without considering human factors, there is only the geometric difference between the research ROI and reference ROI. Therefore, this study artificially introduces contour errors through the following four geometric transformations: translation, scaling, rotation, and sine function transformation based on the reference contour. Then, based on these four transformations, the following objectives were carried out:

1. Study the correlations between the distance-type $\left(\mathrm{HD}, \mathrm{HD}_{\text {mean }}, \mathrm{HD} 95\right)$ and volumetric geometric indices (DSC, Jaccard) and the dosimetric indices (D98\%, D mean, D2\%);

2. Determine the clinical acceptability of using geometric indices to evaluate the accuracy of contouring results;

3. Use dosimetric indices to explore the differences between geometric indices.

\section{Methods}

\section{Contouring}

In order to simplify the experiments, the $\mathrm{C}$-shaped target and Core (OAR) contoured on water phantoms were regarded as the reference ROI (Fig. 1), according to the TG-119 report [17]. The export planning target volume (PTV) and Core's RTStructure from the treatment planning system (TPS) Raystation (Raysearch, Stockholm, Sweden) were used, and then, Python version 3.7.3 software was used to perform the geometric transformations. In order to analyze the contouring errors in detail, translation, scaling, 
rotation (except for the Core), and sine function transformation were simulated for both the C-shaped target and Core.

In this study, the translation transformations were divided into the following three cases: left shift, up shift, and down shift. Based on the location of the reference ROI, at intervals of $1 \mathrm{~mm}$, the data were moved 10 times to each of the left, up, and down directions to obtain the ROI contour. Scaling transformation represents an equidistant expansion or reduction transformation in reference to the position of the ROI. Considering the fast speed of scaling transformation changes, in the patient modeling module of the Raystation planning system, 10 equidistant transformations were performed at $0.5 \mathrm{~mm}$ intervals, excluding the up and down directions. The rotation transformation involved taking the origin of the $\mathrm{CT}$ image coordinates as the rotation center point, using $1^{\circ}$ as the interval, and rotating clockwise 10 times. For the sine function transformation, we extracted the coordinate values $\left(\mathrm{x}_{0}, \mathrm{y}_{0}\right)$ of the reference ROI first, and then, we used the function $y=\sin \omega \mathrm{y}_{0}(\omega=3,4,5,6, \ldots, 12)$ to carry out periodic transformations 10 times with a fixed amplitude.

\section{Geometric indices}

In this study, we chose five widely used geometric indices for the evaluations, including three distancetype indices HD (maximum, mean, 95\%) and two volumetric indices (DSC and Jaccard). The HD (maximum, mean, 95\%) and DSC were calculated by 3DSlicer version 4.10.2 [18], which is open source software. The HD indices calculated by 3DSlicer represent bi-directional distances, and the bi-directional distance is symmetrical; this type of distance is more stable than the unidirectional distance calculated by other methods. We calculated the Jaccard according to its definition, first, by reading the volume parameters of the corresponding ROI from the Raystation planning system, and then, by applying Jaccard $=|A \cap B| /|A \cup B|$, where $A$ represents the reference $R O I$ and $B$ represents the contouring $R O I$.

\section{Dosimetric indices}

In this study, PTV and Core were regarded as the reference ROI. Similarly, the IMRT plan, which meets the requirements for a simple version in the TG-119 report, was taken as the reference plan. The dose of 5000 cGy received by $90 \%$ of the target volume was taken as the prescription, and the dose grid was $2 \mathrm{~mm}$. In order to determine the differences in dosimetric indices caused by different contour errors, the method adopted in this study was to use the existing dose distribution on the reference ROI and overlay it on the contouring ROI [19]. After geometric transformation, RTstructure was imported into the radiotherapy plan of the reference $\mathrm{ROI}$, and then, on the dose distribution, $\mathrm{D} 98 \%, \mathrm{D}_{\text {mean }}$, and $\mathrm{D} 2 \%$ of the PTV and $D_{\text {mean }}$ and D2\% of the Core were obtained. According to the ICRU-83 report [20], these dosimetric indices represent the minimum dose, mean dose, and maximum dose received by the target, and the mean dose and maximum dose received by the organs at risk, respectively. In this study, the dose differences in the three dosimetric indices $\mathrm{D} 98 \%, \mathrm{D}_{\text {mean }}$, and D2\% were calculated and normalized according to their respective clinical goals. Here, $\Delta D=\left(D_{x}\right.$, reference $-D_{x}$, contour $)$, where $x$ represents the type of dosimetric index.

\section{Analysis}


SPSS version 21.0 software (SPSS, Chicago, IL, USA) was used for the statistical analysis. The $\mathrm{R}^{2}$ were used to quantify the correlations between the geometric indices HD (maximum, mean, 95\%), DSC, and Jaccard, and the dosimetric indices D98\%, $\mathrm{D}_{\text {mean }}$, and D2\%. Two-sided p-values were obtained, and pvalues $<0.05$ were considered significant. By using the geometric indices and dose difference diagram, the clinical feasibility of assessing the accuracy of ROI contouring with geometric indices was analyzed.

\section{Results}

The ranges and trends of the distance-type geometric indices of PTV and Core were the same (Fig. 2A-C, $D-F)$. The change trend of $\mathrm{HD}_{\text {mean }}$ was slow, and it was obviously lower than that of the other two indices. HD95 was slightly smaller than HD because HD95 computations eliminate abnormal points and noise points during the calculation process.

The volumetric geometric indices DSC and Jaccard of PTV changed more slowly than those of the Core (Fig. 2C, F), and the declining rate of Jaccard was also slower than that of DSC. From the definition of the volumetric geometric index, this phenomenon can be explained. For a transformation of the same distance, a larger surface area of the ROI will correspond to a larger volumetric geometric index, and the DSC will be greater than Jaccard.

Linear regression analysis was carried out on the geometric indices and dosimetric indices (Fig. 3A, B and $3 C$, D show the results for the PTV and Core, respectively). Except for the sine function transformation $\left(R^{2}: 0.04-0.023, p>0.05\right)$, the other three geometric indices of the PTV had strong correlations with the dosimetric indices $D 98 \%$ and $D_{\text {mean }}\left(R^{2}: 0.689-0.988\right), 80 \%$ of which were strongly correlated with a $p<$ 0.001 . D2\% was not included in the correlation analysis because of its small variation range. The correlation results for the other geometric transformations in the Core were similar to those in the PTV except for the down shift transformation (the correlations between the geometric indices and $\mathrm{D}_{\text {mean }}$ and D2\% were weak with a $p>0.05$, which can be regarded as uncorrelated).

Assuming that there is a reference ROI, differences in dosimetric indices of the radiotherapy plan are the most effective method to test the clinical contouring results. Therefore, in this study we investigated whether the geometric indices can be used to effectively predict the changes in dosimetric indices.

Figures 4 and 5 show the relationships between the geometric indices of the PTV and Core and the dose difference. In a certain range, the smaller the distance-type geometric index value was, i.e., the closer to 0 , the smaller the dose difference was; meanwhile, the larger the volumetric geometric index value was, i.e., the closer to 1 , the smaller the dose difference was. Clinically, it is generally accepted that a dose difference within $\pm 5 \%$ is acceptable, but it can be seen from Figs. 4 and 5 that within the acceptable dose change range, the relationships between the geometric indices and dose difference were not monotonous and consistent.

\section{Discussion}


Many studies have shown that although it is important to quantify the degree of variation or uncertainty of the contouring, it is more important to determine the dose difference and clinical impact $[10,11,14$, 21]. In earlier work, van Rooij et al. [21] studied the accuracy of automatic delineation of dangerous head and neck organs based on deep learning techniques while using geometric indices and dosimetric indices, and they analyzed the correlation between the geometric index SDC (mean value of the DSC) and dose difference. That study found that there was a weak correlation between the SDC and $\triangle D$ for all of the organs in danger through automatic segmentation, $r=-0.24, P=0.002$, but the correlation was not specific to a certain organ in danger or a certain patient. This is similar to the results described in this study. We found that the geometric indices obtained by geometric transformation were significantly correlated with the dosimetric indices, but with a certain geometric transformation form, the situation was different.

In this study, there was a strong and significant correlation between the geometric indices and dosimetric indices in the translation, scaling, and rotation transformations of the PTV, but the results for the sine function transformation were not significant or weak; the correlation for the PTV down shift transformation was strong, but the correlation for the Core down shift transformation was weak and not significant. This indicates that there is only a statistically significant overall correlation between the geometric indices and dosimetric indices, but this correlation is not consistent for the different forms of geometric transformations and organ types. This shows that the correlation between geometric indices and dosimetric indices can be affected by many factors, such as the method of geometric transformation, the relative positions of the target and organs at risk, the shape and size of the ROI, and the constraint goals of the radiotherapy plan.

In this study, the correlation coefficient obtained with the PTV up shift transformation was lower than that of the other two translation transformation methods. In order to avoid high doses of radiation, the high dose area of the PTV should ideally be far away from the OAR in the plan design. Therefore, when the up shift transformation occurs in this area, the minimum dose (D98\%) of the target basically does not change, thus resulting in a weaker correlation coefficient. This is in contrast to the study by Lim et al. [10], which found that the correlation between geometric indices and dosimetric indices was affected by the goals of the treatment plan. Feng et al. [22] considered that the contour changes of oropharyngeal carcinoma OAR had little effect on the dose, but Nelms et al. [23] believed that this had a great effect on the dose. The systematic simulation research in this study indicates that there is no definite correlation between geometric indices and dosimetric indices. The weak correlation and inconsistent relationship between geometric indices and dosimetric indices also help explain the contradictory content in the above literature.

Nowadays, most contour research only uses geometric indices to evaluate the acceptability of contouring results. With the aim of using an objective evaluation method (for geometric indices), it is generally believed that a DSC value greater than 0.7 means the contouring result is clinically acceptable [24-27], while for distance-type geometric indices $\left(\mathrm{HD}, \mathrm{HD}_{\text {mean }}, \mathrm{HD} 95\right)$, smaller values mean that the contouring result is clinically acceptable, and the closer the value is to zero, the better the contouring result is. 
However, this statement is ambiguous. The article about the study of automatic segmentation of OARs showed that an (Liver) HD $=15.770 \pm 1.0 \mathrm{~mm}$ had a high accuracy [27], while another article indicated that an $\mathrm{HD}=37.7 \pm 13.8 \mathrm{~mm}$ also showed a high accuracy [28]. Although the former value is smaller than the latter, due to the lack of a standard reference, which one has the higher accuracy cannot be clearly stated. This makes HD results not comparable and not readily distinguishable.

In this study, we found that the changes in geometric indices and dosimetric indices did not correspond to each other. It can be seen from Fig. 2 that the $\mathrm{HD}_{\text {mean }}$ changed rapidly during the PTV translation, rotation, and scaling. When $\mathrm{HD}_{\text {mean }}$ was equal to $5 \mathrm{~mm}$, the dose difference was not clinically acceptable. The distance-type geometric indices of scaling transformation were consistent, and the HD, HDmean, and HD95 were all indistinguishable from scaling differences. The distance-type geometric indices of the sine function transformation were very small, and the corresponding dose differences were also very small and all within the clinical acceptable range; although the distance-type geometric indices of up shift transformation exceeded $8 \mathrm{~mm}$, the dose differences were still within the acceptable range. The geometric indices of left shift, up shift, and sine function transformation for dangerous organs were within the range of less than $2 \mathrm{~mm}$, and D2\% was within $5 \%$ of the dose difference, but the D2\% of the up shift and scale transformation was outside the range, which shows that it is not reliable to evaluate the contour results only by geometric indices, which is consistent with the opinions of Kaderka et al. [11]. When the values of DSC and Jaccard are between 0.5 and 0.7 , the corresponding dose difference is very small, and the difference is also clinically acceptable, which also proves that it is unreliable to set 0.7 as the clinically acceptable threshold of the DSC [29].

Beasley et al. [30] also reported that when measured with a suitable spatial metric, the higher the geometric accuracy of the contour, the smaller the dose difference should therefore be reflected, and vice versa. Our research showed that when the geometric index was within the acceptable threshold range, the corresponding dose difference can be clinically unacceptable, or when the geometric index was beyond the acceptable threshold range, the corresponding dose difference can be clinically acceptable. Although geometric indices reflect the difference between a specific contour and reference contour, the inconsistent response between geometric indices and dosimetric indices makes it meaningless to evaluate the clinical acceptability of contour results only by geometric indices, and geometric indices cannot predict the clinical dose difference.

Our research systematically introduced the geometric transformation of translation, scaling, rotation and sine function. Under the reference dose distribution, we analyzed the feasibility of clinical evaluation of geometric indices according to the geometric transformation type. And we discussed the influence of position distance change caused by multiple geometric errors of a structure between geometric and dosimetric indices, but the actual relationship between them is very complex. In addition, as this study is a simulation experiment, we should further explore the structures' size and properties of actual patient data.

\section{Conclusion}


At present, there is a lack of consistency and guidance in evaluations of the ROI contour accuracy, and there should be a normative framework. Through the analysis of geometric and dosimetric indices, we found that the differences between the geometric indices and dosimetric indices were not consistent, which makes the accuracy of only using the geometric indices to evaluate the results of contouring defective. The clinical acceptability of contouring results cannot be judged by geometric indices alone. Results are affected by the shape, size, position, and other attributes of the contoured structure, and the selection of geometric indices should not be arbitrary. Therefore, we suggest that dosimetric indices should be added to evaluations of the accuracy of the results of delineation, which can be helpful for explaining the clinical dose response relationship of delineation more comprehensively, accurately.

\section{Abbreviations}

IMRT: intensity-modulated radiotherapy; AAPM: the American Association of Physicists in Medicine; TG119 report: The report of Task Group 119 of the AAPM; PTV: planning target volume; OAR: organ at risk; VMAT: volumetric modulated arc radiotherapy; CT: computed tomography; ROI: the region of interest; HD: the maximum Hausdorff distances; HDmean: the mean Hausdorff distances; HD95: the 95\% Hausdorff distances; DSC: Dice-similarity coefficient; RT: radiotherapy; TPS: the treatment planning system; SDC: mean value of the Dice-similarity coefficient

\section{Declarations}

\section{Ethics approval and consent to participate}

Not applicable.

\section{Consent for publication}

Not applicable.

\section{Availability of data and materials}

Please contact author for data requests.

\section{Competing interests}

The authors declare that they have no competing interests.

\section{Funding}

This work was supported by the National Natural Science Foundation of China (Grant No. 81472807).

\section{Authors' contributions}


LXX and GJL analyzed the data and drafted the manuscript. QX, ZBL and XBZ performed the experiments. LC and ZYH collected data. SB designed this study, interpreted the results and revised the manuscript. All authors read and approved the final manuscript.

\section{Acknowledgements}

Not applicable.

\section{References}

1. Brouwer CL, Steenbakkers RJ, van den Heuvel E, Duppen JC, Navran A, Bijl HP, Chouvalova O, Burlage FR, Meertens $\mathrm{H}$, Langendijk JA, van T Veld AA. 3D Variation in delineation of head and neck organs at risk. RADIAT ONCOL. 2012;32(7).

2. Brouwer CL, Steenbakkers RJHM, Bourhis J, Budach W, Grau C, Grégoire V, van Herk M, Lee A, Maingon P, Nutting C, O Sullivan B, Porceddu SV, Rosenthal DI, Sijtsema NM, Langendijk JA. CTbased delineation of organs at risk in the head and neck region: DAHANCA, EORTC, GORTEC, HKNPCSG, NCIC CTG, NCRI, NRG Oncology and TROG consensus guidelines. RADIOTHER ONCOL. 2015;117(1):83-90.

3. Khoo VS, Adams EJ, Saran F, Bedford JL, Perks JR, Warrington AP, Brada M. A Comparison of clinical target volumes determined by $\mathrm{CT}$ and MRI for the radiotherapy planning of base of skull meningiomas. Int J Radiat Oncol Biol Phys. 2000;46(5):1309-1317.

4. Papiez L, Langer M. On probabilistically defined margins in radiation therapy. PHYS MED BIOL. 2006;51(16):3921-3939.

5. La Macchia M, Fellin F, Amichetti M, Cianchetti M, Gianolini S, Paola V, Lomax AJ, Widesott L. Systematic evaluation of three different commercial software solutions for automatic segmentation for adaptive therapy in head-and-neck, prostate and pleural cancer. Radiation oncology (London, England). 2012;7(1):160.

6. Lim JY, Leech M. Use of auto-segmentation in the delineation of target volumes and organs at risk in head and neck. ACTA ONCOL. 2016;55(7):799-806.

7. Chaney E, Ibbott G, Hendee WR. Methods for image segmentation should be standardized and calibrated. MED PHYS. 2005;32(12):3507-3510.

8. Zhu M, Bzdusek K, Brink C, Eriksen JG, Hansen O, Jensen HA, Gay HA, Thorstad W, Widder J, Brouwer CL, Steenbakkers RJHM, Vanhauten HAM, Cao JQ, McBrayne G, Patel SH, Cannon DM, Hardcastle N, Tomé WA, Guckenberg M, Parikh PJ. Multi-institutional Quantitative Evaluation and Clinical Validation of Smart Probabilistic Image Contouring Engine (SPICE) Autosegmentation of Target Structures and Normal Tissues on Computer Tomography Images in the Head and Neck, Thorax, Liver, and Male Pelvis Areas. International Journal of Radiation Oncology*Biology*Physics. 2013;87(4):809-816. 
9. Taha AA, Hanbury A. Metrics for evaluating 3D medical image segmentation: analysis, selection, and tool. BMC MED IMAGING. 2015;15(1).

10. Lim TY, Gillespie E, Murphy J, Moore KL. Clinically Oriented Contour Evaluation Using Dosimetric Indices Generated From Automated Knowledge-Based Planning. International Journal of Radiation Oncology*Biology*Physics. 2019;103(5):1251-1260.

11. Kaderka R, Gillespie EF, Mundt RC, Bryant AK, Sanudo-Thomas CB, Harrison AL, Wouters EL, Moiseenko V, Moore KL, Atwood TF, Murphy JD. Geometric and dosimetric evaluation of atlas based auto-segmentation of cardiac structures in breast cancer patients. RADIOTHER ONCOL. 2019;131:215-220.

12. Jameson MG, Holloway LC, Vial PJ, Vinod SK, Metcalfe PE. A review of methods of analysis in contouring studies for radiation oncology. J Med Imaging Radiat Oncol. 2010;54(5):401-410.

13. Hanna GG, Hounsell AR, O Sullivan JM. Geometrical Analysis of Radiotherapy Target Volume Delineation: a Systematic Review of Reported Comparison Methods. CLIN ONCOL-UK. 2010;22(7):515-525.

14. Vinod SK, Jameson MG, Min M, Holloway LC. Uncertainties in volume delineation in radiation oncology: A systematic review and recommendations for future studies. RADIOTHER ONCOL. 2016;121(2):169-179.

15. Tsuji SY, Hwang A, Weinberg V, Yom SS, Quivey JM, Xia P. Dosimetric Evaluation of Automatic Segmentation for Adaptive IMRT for Head-and-Neck Cancer. International Journal of Radiation Oncology*Biology*Physics. 2010;77(3):707-714.

16. Kieselmann JP, Kamerling CP, Burgos N, Menten MJ, Fuller CD, Nill S, Cardoso MJ, Oelfke U. Geometric and dosimetric evaluations of atlas-based segmentation methods of MR images in the head and neck region. PHYS MED BIOL. 2018;63(14):145007.

17. Ezzell GA, Burmeister JW, Dogan N, LoSasso TJ, Mechalakos JG, Mihailidis D, Molineu A, Palta JR, Ramsey CR, Salter BJ, Shi J, Xia P, Yue NJ, Xiao Y. IMRT commissioning: Multiple institution planning and dosimetry comparisons, a report from AAPM Task Group 119. MED PHYS. 2009;36(11):53595373.

18. Pinter C, Lasso A, Wang A, Jaffray D, Fichtinger G. SlicerRT: Radiation therapy research toolkit for 3D Slicer. MED PHYS. 2012;39(10):6332-6338.

19. Conson M, Cella L, Pacelli R, Comerci M, Liuzzi R, Salvatore M, Quarantelli M. Automated delineation of brain structures in patients undergoing radiotherapy for primary brain tumors: From atlas to dose-volume histograms. RADIOTHER ONCOL. 2014;112(3):326-331.

20. Prescribing, Recording, and Reporting Photon-Beam Intensity-Modulated Radiation Therapy (IMRT): Contents. Journal of the ICRU. 2010;10(1):3.

21. van Rooij W, Dahele M, Ribeiro Brandao H, Delaney AR, Slotman BJ, Verbakel WF. Deep LearningBased Delineation of Head and Neck Organs at Risk: Geometric and Dosimetric Evaluation. International Journal of Radiation Oncology*Biology*Physics. 2019;104(3):677-684. 
22. Feng M, Demiroz C, Vineberg KA, Eisbruch A, Balter JM. Normal Tissue Anatomy for Oropharyngeal Cancer: Contouring Variability and Its Impact on Optimization. International Journal of Radiation Oncology*Biology*Physics. 2012;84(2):e245-e249.

23. Nelms BE, Tomé WA, Robinson G, Wheeler J. Variations in the Contouring of Organs at Risk: Test Case From a Patient With Oropharyngeal Cancer. International Journal of Radiation Oncology*Biology*Physics. 2012;82(1):368-378.

24. Mattiucci GC, Boldrini L, Chiloiro G, D Agostino GR, Chiesa S, De Rose F, Azario L, Pasini D, Gambacorta MA, Balducci M, Valentini V. Automatic delineation for replanning in nasopharynx radiotherapy: What is the agreement among experts to be considered as benchmark? ACTA ONCOL. 2013;52(7):1417-1422.

25. Sharp G, Fritscher KD, Pekar V, Peroni M, Shusharina N, Veeraraghavan H, Yang J. Vision 20/20: Perspectives on automated image segmentation for radiotherapy. MED PHYS. 2014;41(5):50902.

26. Eldesoky AR, Yates ES, Nyeng TB, Thomsen MS, Nielsen HM, Poortmans P, Kirkove C, Krause M, Kamby C, Mjaaland I, Blix ES, Jensen I, Berg M, Lorenzen EL, Taheri-Kadkhoda Z, Offersen BV. Internal and external validation of an ESTRO delineation guideline - dependent automated segmentation tool for loco-regional radiation therapy of early breast cancer. RADIOTHER ONCOL. 2016;121(3):424-430.

27. Liang F, Qian P, Su K, Baydoun A, Leisser A, Van Hedent S, Kuo J, Zhao K, Parikh P, Lu Y, Traughber BJ, Muzic RF. Abdominal, multi-organ, auto-contouring method for online adaptive magnetic resonance guided radiotherapy: An intelligent, multi-level fusion approach. ARTIF INTELL MED. 2018;90:34-41.

28. Wittenstein O, Hiepe P, Sowa LH, Karsten E, Fandrich I, Dunst J. Automatic image segmentation based on synthetic tissue model for delineating organs at risk in spinal metastasis treatment planning. STRAHLENTHER ONKOL. 2019;195(12):1094-1103.

29. Mattiucci GC, Boldrini L, Placidi L, Azario L, Dinapoli N, Chiloiro G, Pasini D, Piccari D, Gambacorta MA, Balducci M, Mantini G, Valentini V. Beyond geometrical overlap: a Dosimetrical Evaluation of automated volumes Adaptation (DEA) in head and neck replanning. Technical Innovations \& Patient Support in Radiation Oncology. 2017;3-4:1-6.

30. Beasley WJ, McWilliam A, Aitkenhead A, Mackay RI, Rowbottom CG. The suitability of common metrics for assessing parotid and larynx autosegmentation accuracy. J APPL CLIN MED PHYS. 2016;17(2):41-49.

\section{Figures}




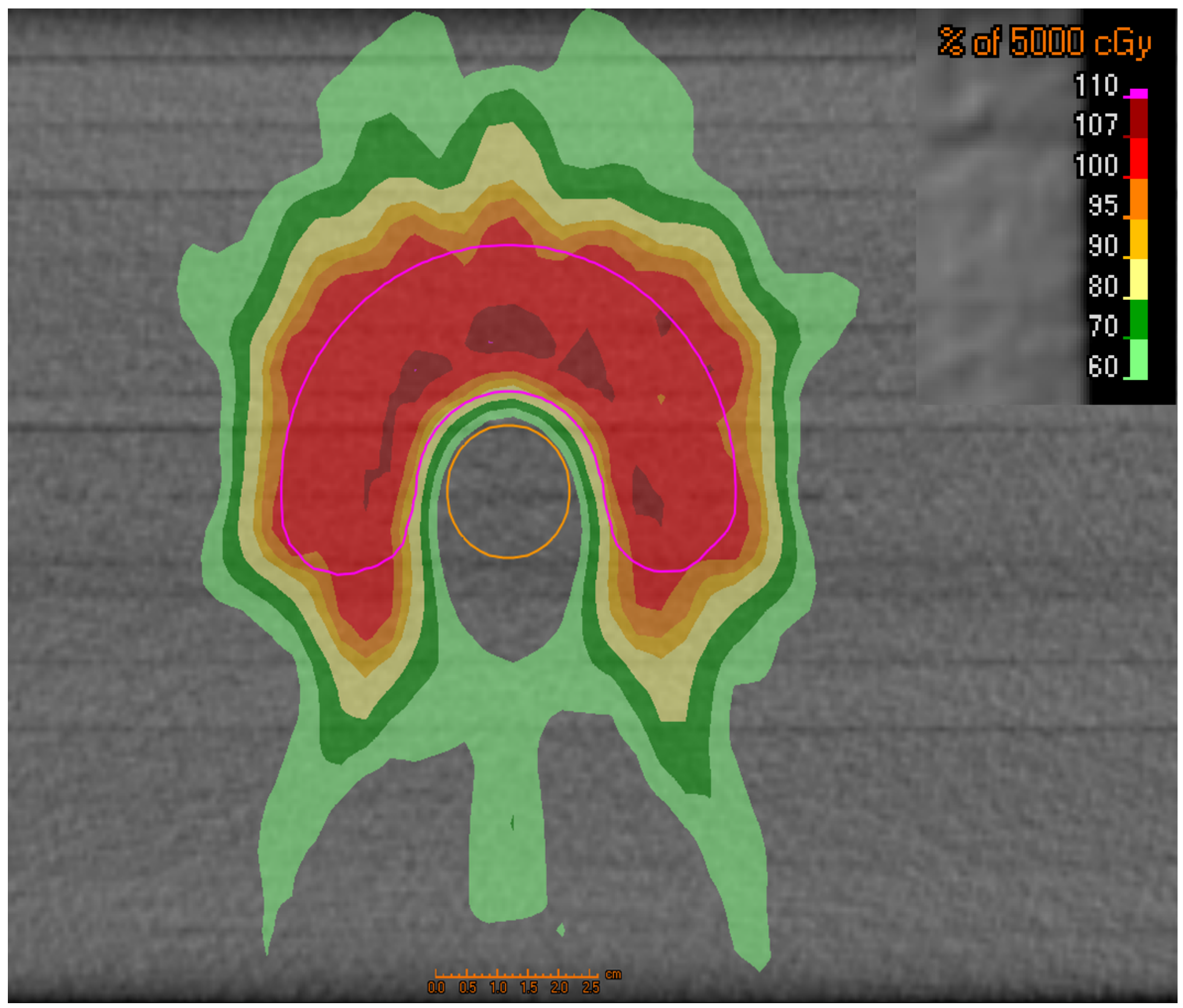

\section{Figure 1}

CT image showing the relative position and dose distribution for the C-shaped target (PTV) and organ at risk (Core). 
A

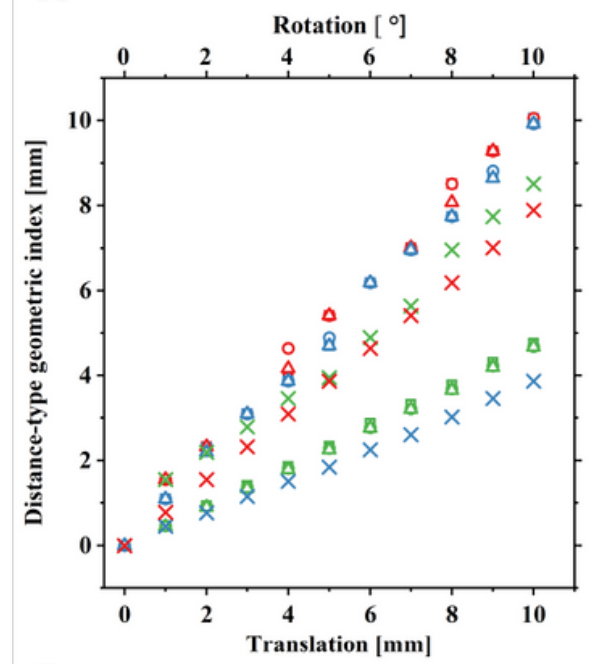

$\mathrm{D}$

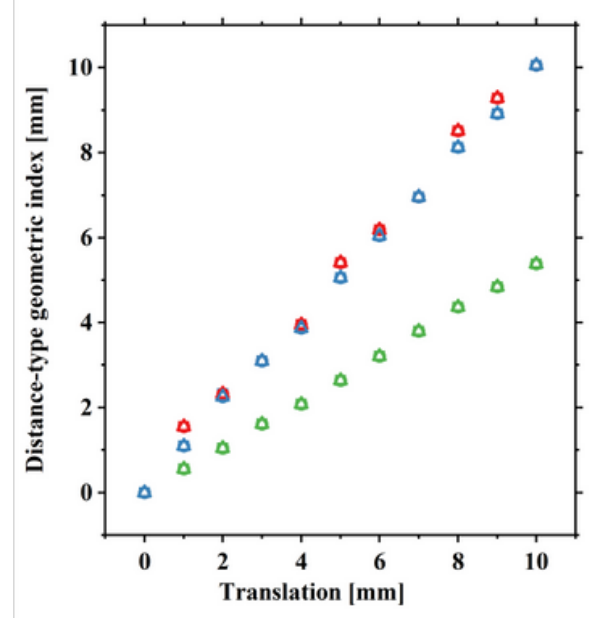

$\begin{array}{lllllll}\square & \text { L-S-HD } & \circ & \text { U-S-HD } & \Delta & \text { D-S-HD } & \times \text { R-HD } \\ \square & \text { L-S-HDmean } & \circ & \text { U-S-HDmean } & \Delta & \text { D-S-HDmean } & \times \text { R-HDmean } \\ \square & \text { L-S-HD95 } & \circ & \text { U-S-HD95 } & \Delta & \text { D-S-HD95 } & \times \text { R-HD95 }\end{array}$
B

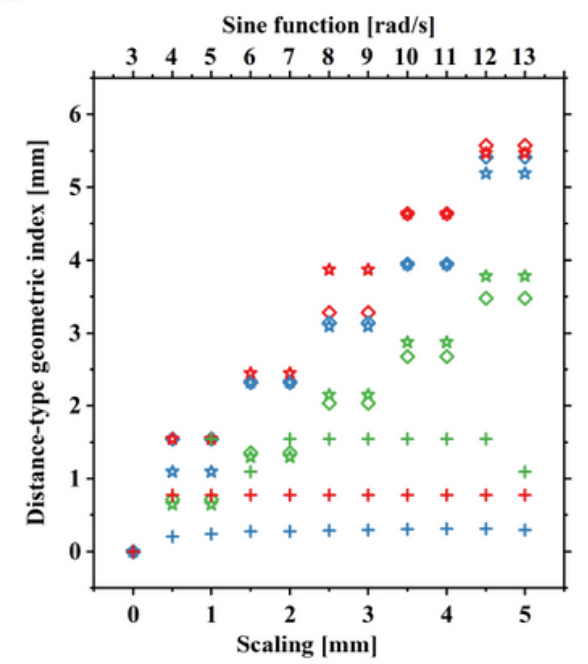

$\mathrm{E}$
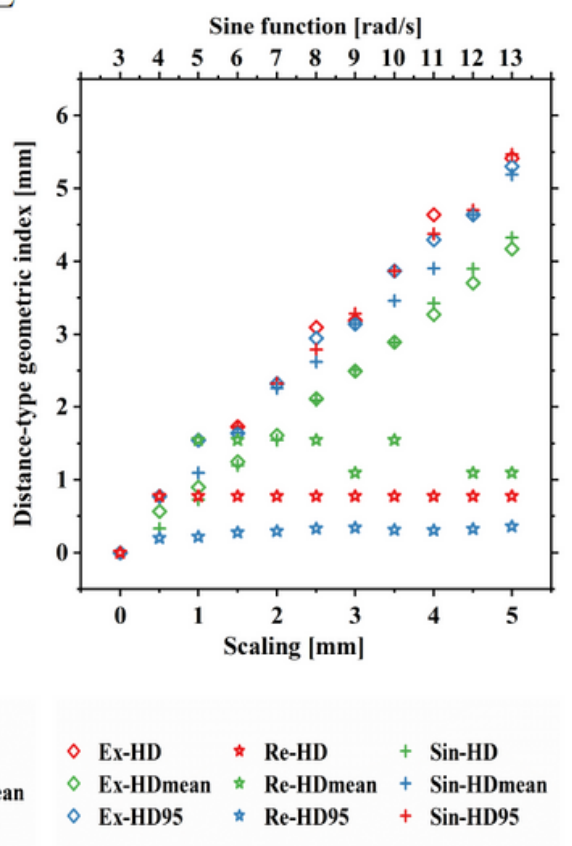

$\mathrm{C}$

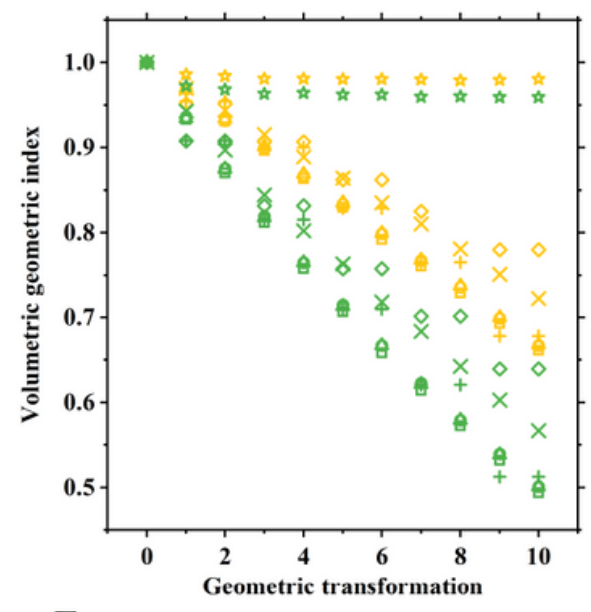

F

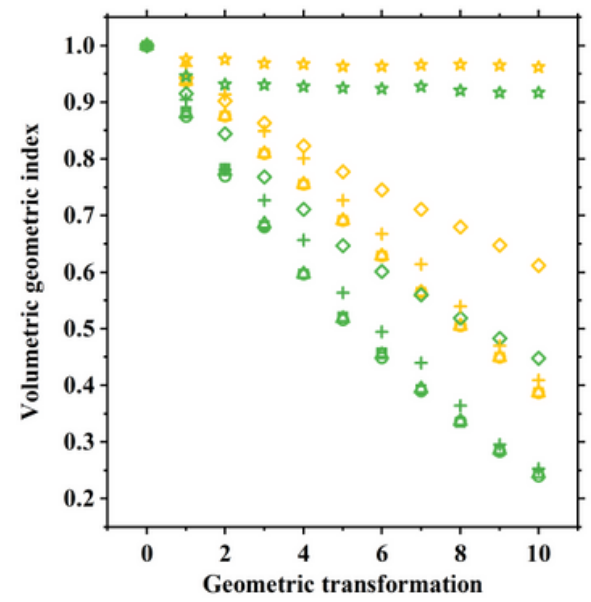

L-S-DSC $\triangle$ U-S-DSC $\triangle$ D-S-DSC $\triangleleft$ Ex-DSC

$\checkmark$ L-S-Jacerd $\diamond$ U-S-Jacerd $\triangle$ D-S-Jaccard $\triangleleft$ Ex-Jaccard

+ Re-DSC $\times$ R-DSC $*$ Sin-DSC

+ Re-Jaccard X R-Jaccard * Sin-Jaceard

\section{Figure 2}

(A-C) and (D-F) depict the distance-type geometric indices (HD, HDmean, HD95) and volumetric geometric indices (DSC, Jaccard) after geometric transformation of the C-shaped target (PTV) and organ at risk (Core), respectively. L-S-HD $=$ HD for left shift transformations; L-S-HDmean $=$ HDmean for left shift transformations; L-S-HD95 = HD95 for left shift transformations; U-S-HD = HD for up shift transformations; U-S-HDmean = HDmean for up shift transformations; U-S-HD95 $=$ HD95 for up shift transformations; D-S-HD = HD for down shift transformations; D-S-HDmean = HDmean for down shift transformations; D-S-HD95 = HD95 for down shift transformations; R-HD = HD for rotation transformations; R-HDmean = HDmean for rotation transformations; R-HD95 = HD95 for rotation transformations; Ex-HD = HD for expansion transformations; Ex-HDmean = HDmean for expansion transformations; Ex-HD95 = HD95 for expansion transformations; Re-HD = HD for reduction transformations; Re-HDmean $=$ HDmean for reduction transformations; Re-HD95 $=$ HD95 for reduction 
transformations; Sin-HD = HD for sine function transformations; Sin-HDmean = HDmean for sine function transformations; Sin-HD95 = HD95 for sine function transformations; L-S-DSC = DSC for left shift transformations; L-S-Jaccard = Jaccard for left shift transformations; U-S-DSC = DSC for up shift transformations; U-S-Jaccard = Jaccard for up shift transformations; D-S-DSC = DSC for down shift transformations; D-S-Jaccard = Jaccard for down shift transformations; R-DSC = DSC for rotation transformations; R-Jaccard = Jaccard for rotation transformations; Ex-DSC = DSC for expansion transformations; Ex-Jaccard = Jaccard for expansion transformations; Re-DSC = DSC for reduction transformations; Re-Jaccard = Jaccard for reduction transformations; Sin-DSC = DSC for sine function transformations; Sin-Jaccard = Jaccard for sine function transformations. 
A

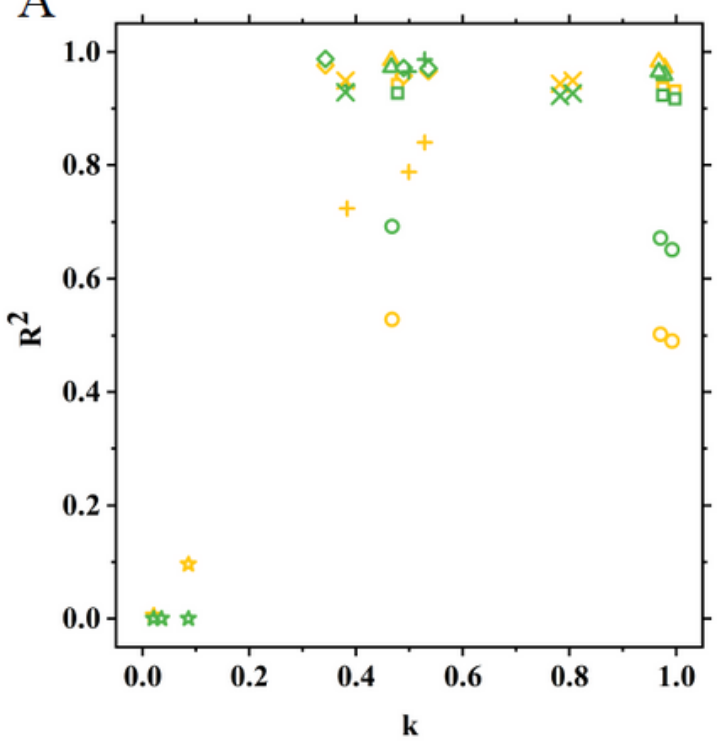

B

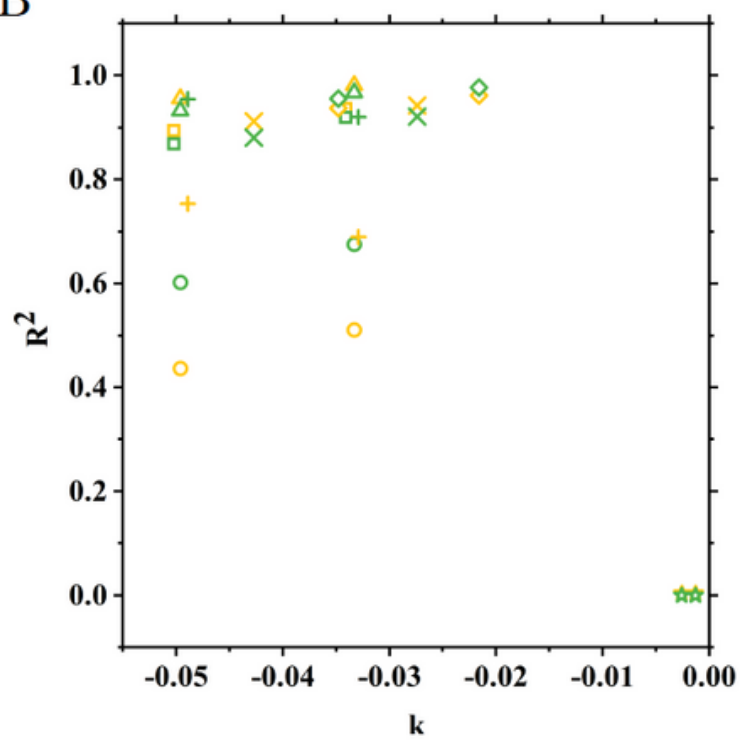

ㄴ-S-D98\% $\odot$ U-S-D98\% $\triangle$ D-S-D98\% $\diamond$ Ex-D98\% + Re-D98\% $\%$ R-D98\% $*$ Sin-D98\%

$\checkmark$ L-S-Dmean $\diamond$ U-S-Dmean $\Delta$ D-S-Dmean $\diamond$ Ex-Dmean + Re-Dmean $\times$ R-Dmean $\star$ Sin-Dmean

$\mathrm{C}$

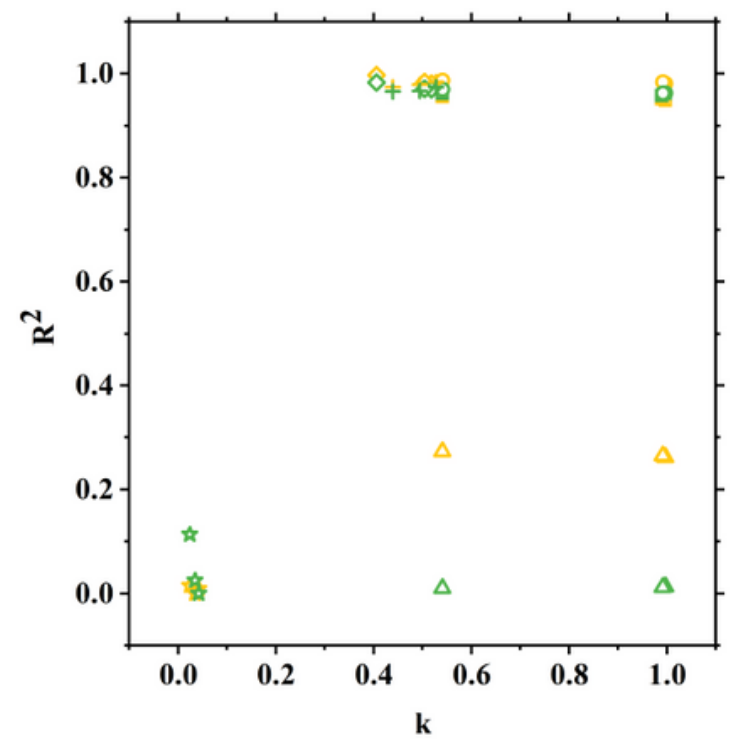

L-S-Dmean $\circ$ U-S-Dmean $\triangle$ D-S-Dmean

ㄴ-S-D2\% $\quad \circ \quad$ U-S-D2\% $\quad \Delta$ D-S-D2\%
$\mathrm{D}$

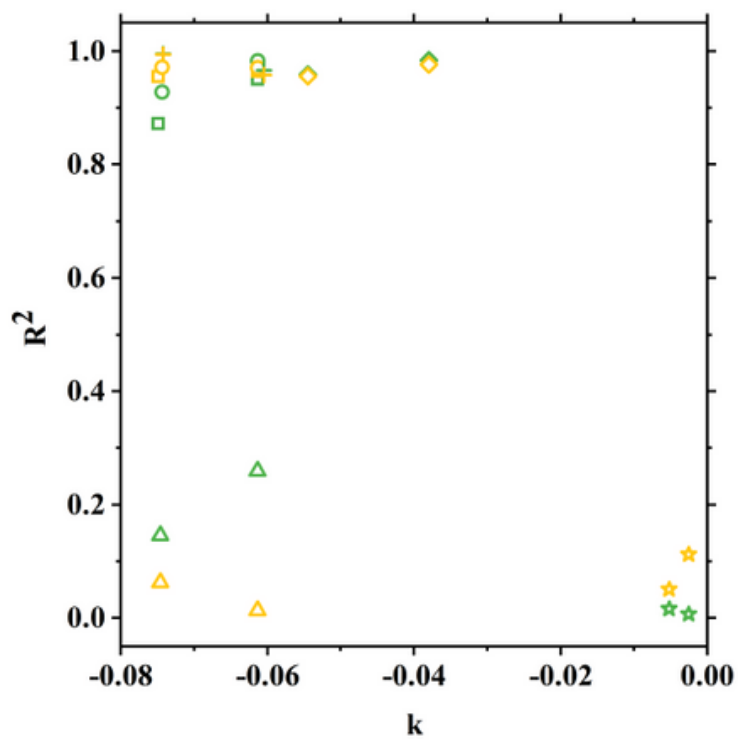

\section{Figure 3}

(A, B), (C, D) represent the correlations between the geometric indices and dosimetric indices for the PTV and Core, respectively. The abscissa represents the slope of the transformation trend for the geometric indices, and the ordinate is the correlation coefficient of the linear regression analysis. L-S-D98\% = D98\% for left shift transformations; L-S-Dmean = Dmean for left shift transformations; U-S-D98\% = D98\% for up shift transformations; U-S-Dmean = Dmean for up shift transformations; D-S-D98\% = D98\% for down shift 
transformations; D-S-Dmean = Dmean for down shift transformations; Ex-D98\% = D98\% for expansion transformations; Ex-Dmean = Dmean for expansion transformations; Re-D98\% = D98\% for reduction transformations; Re-Dmean = Dmean for reduction transformations; $\mathrm{R}-\mathrm{D} 98 \%=\mathrm{D} 98 \%$ for rotation transformations; R-Dmean = Dmean for rotation transformations; Sin-D98\% = D98\% for sine function transformations; Sin-Dmean = Dmean for sine function transformations; L-S-D2\% = D2\% for left shift transformations; U-S-D2\% = D2\% for up shift transformations; D-S-D2\% = D2\% for down shift transformations; Ex-D2\% = D2\% for expansion transformations; Re-D2\% = D98\% for reduction transformations; Sin-D2\% = D2\% for sine function transformations. 


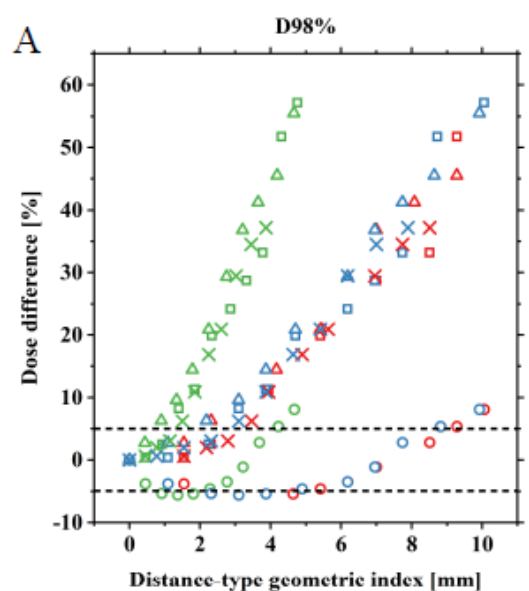

$\mathrm{D}$
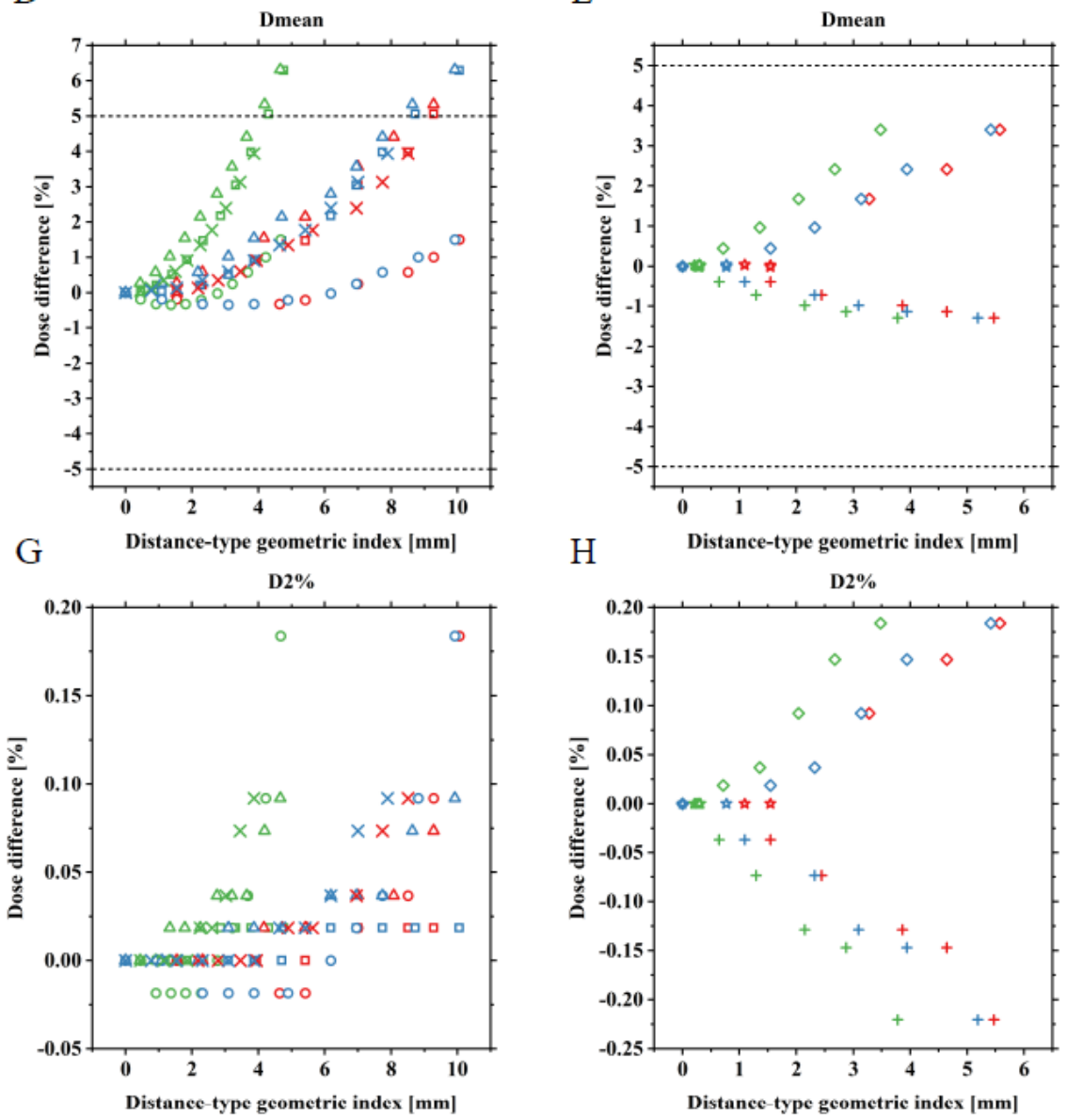

$\mathrm{E}$
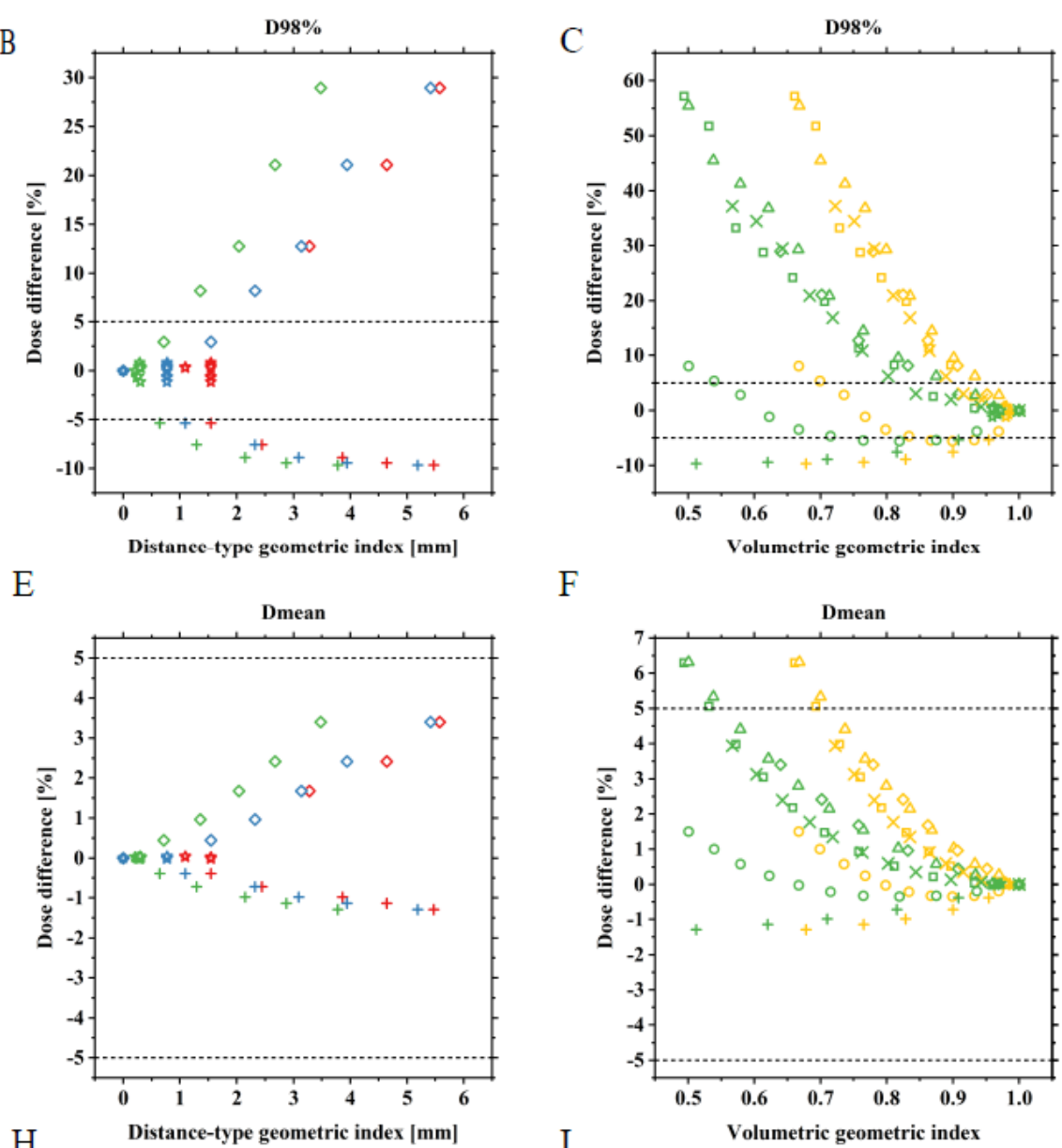

F

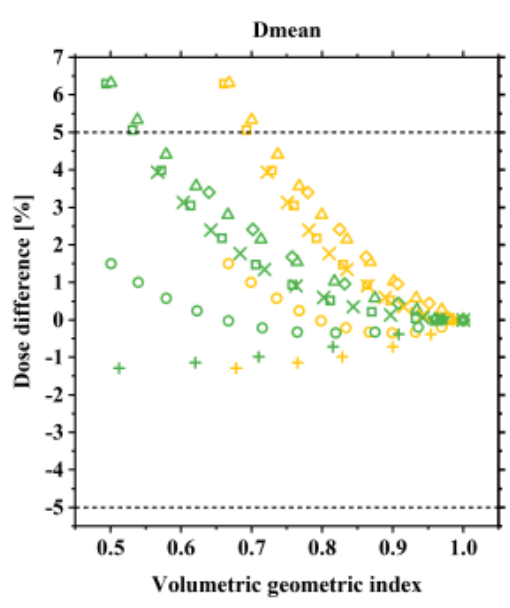

I
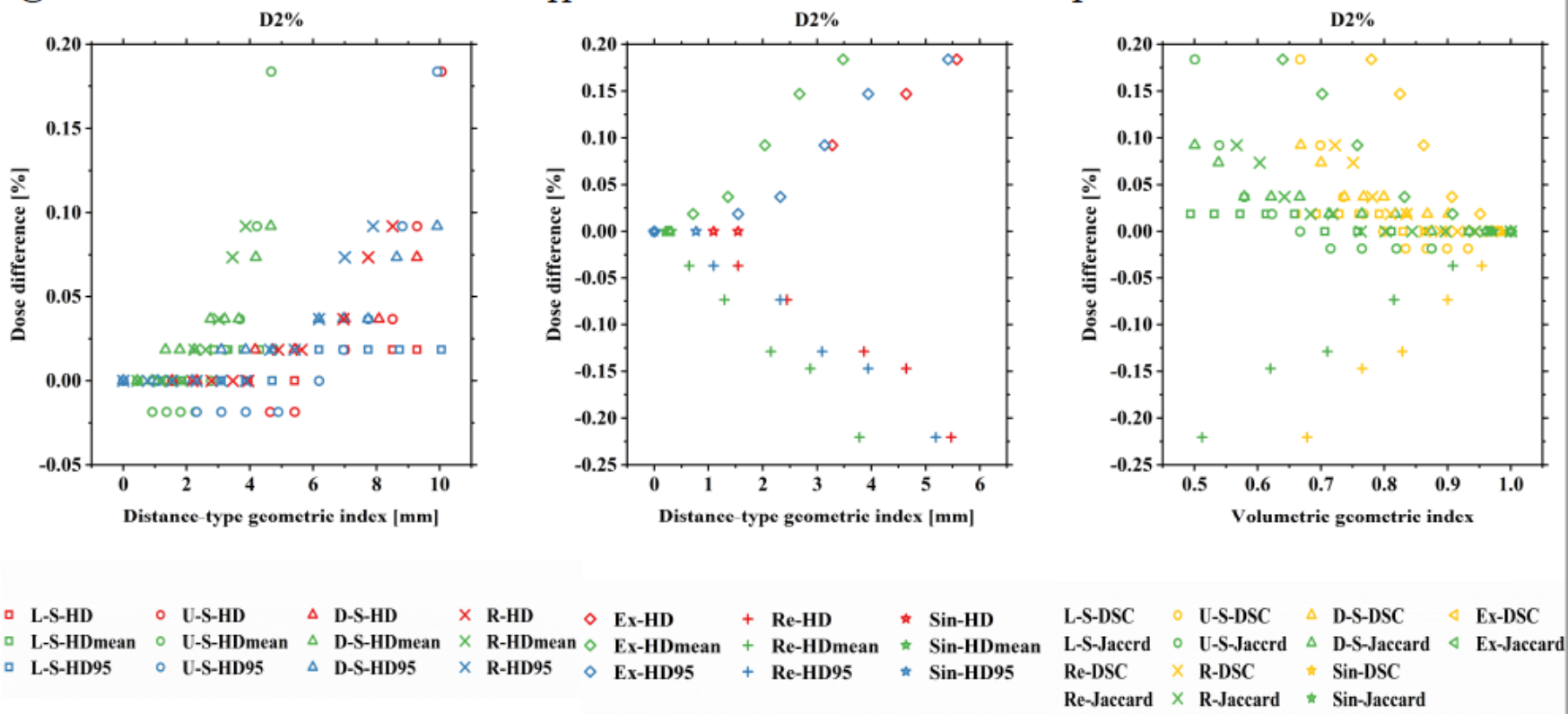

Figure 4

The relationship between the geometric indices of PTV and the dose difference; the dotted line shows the acceptable dose tolerance of $\pm 5 \%$. 


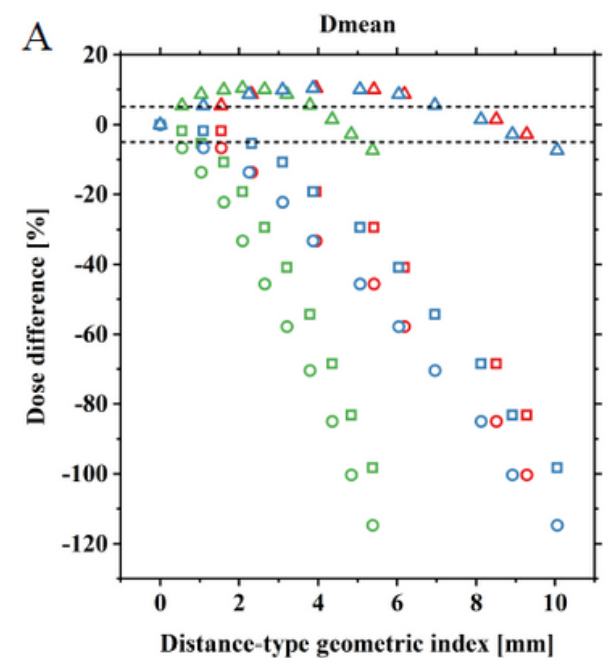

$\mathrm{D}$

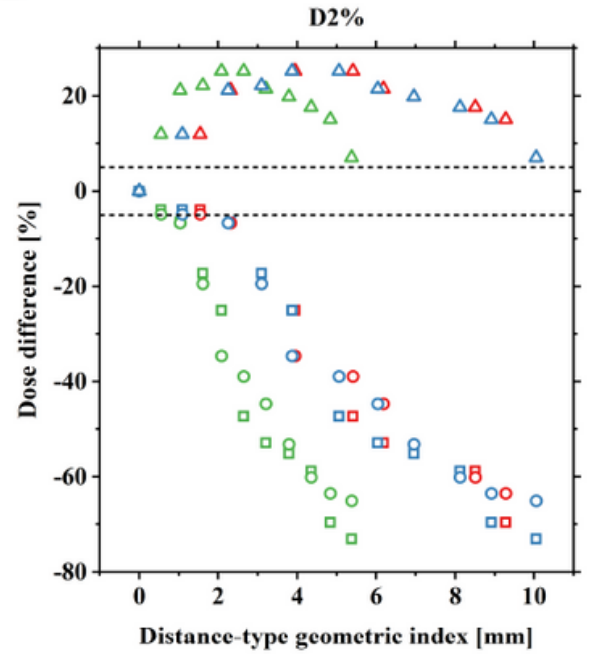

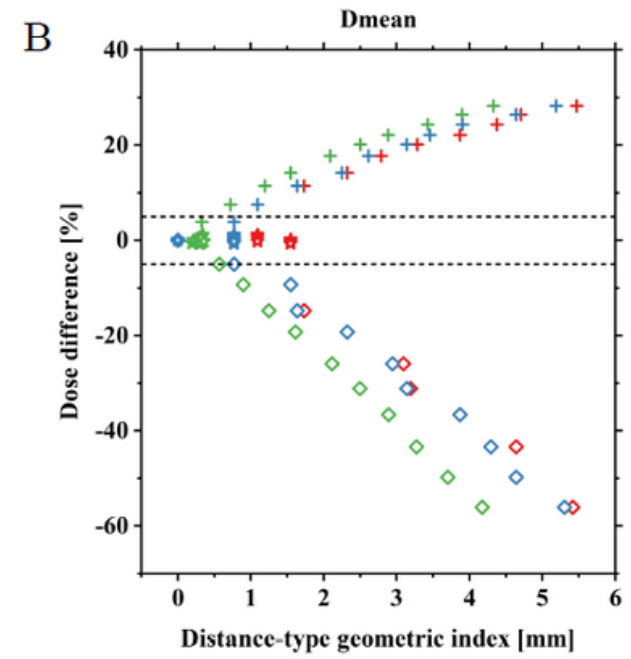

$\mathrm{E}$
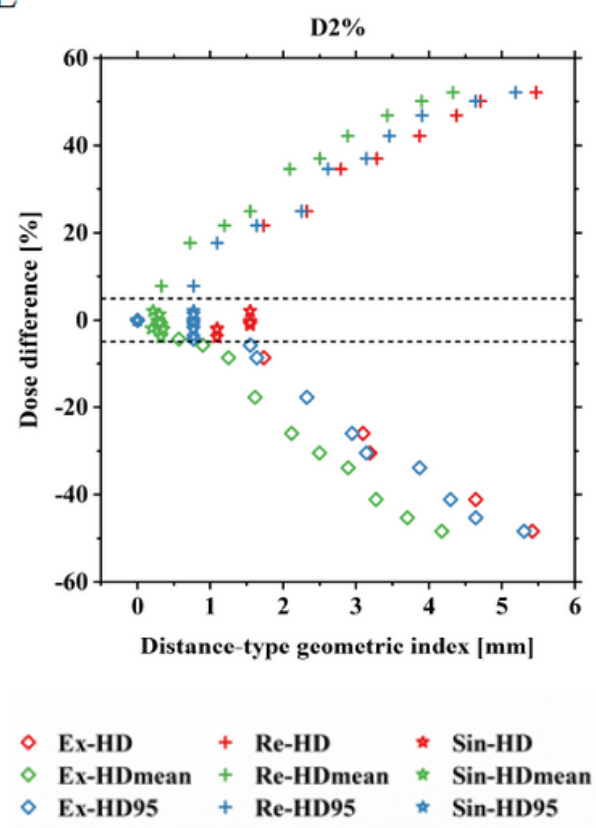

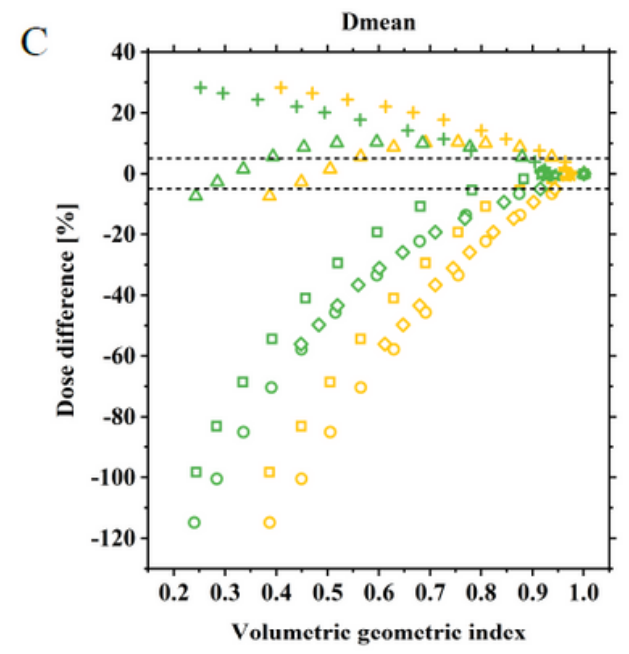

$\mathrm{F}$

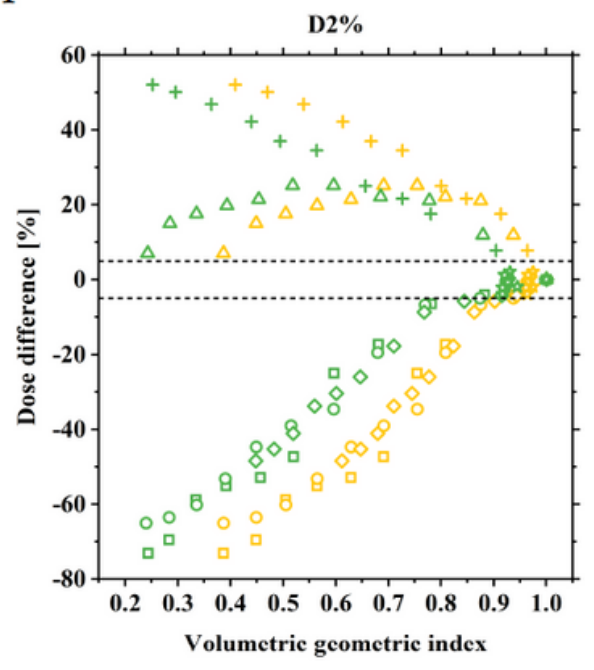

$\begin{array}{llllll}\square & \text { L-S-DSC } & \circ & \text { U-S-DSC } & \Delta & \text { D-S-DSC } \\ \square & \text { L-S-Jacerd } & \circ & \text { U-S-Jacerd } & \Delta & \text { D-S-Jaceard } \\ & \text { Ex-DSC } & + \text { Re-DSC } & \Rightarrow & \text { Sin-DSC } \\ \diamond & \text { Ex-Jaccard } & + \text { Re-Jaccard } & \Rightarrow & \text { Sin-Jaccard }\end{array}$

\section{Figure 5}

The relationship between the geometric indices of Core and the dose difference; the dotted line shows the acceptable dose tolerance of $\pm 5 \%$. 